potential mechanistic link to elite athlete infection susceptibility.

Please refer to page A190 for declarations of interest related to this abstract.

\section{S95 TRANSCRIPTIONAL SIGNATURES OF BLOOD OUTGROWTH ENDOTHELIAL CELLS FROM PATIENTS WITH PULMONARY ARTERIOVENOUS MALFORMATIONS AND HEREDITARY HAEMORRHAGIC TELANGIECTASIA}

${ }^{1}$ ME Bernabeu Herrero, ${ }^{1} A$ Bielowka, ${ }^{1} D$ Patel, ${ }^{2} S$ Srikaran, ${ }^{1} \mathrm{P}$ Chaves Guerrero, ${ }^{1} \mathrm{M}$ Noseda, ${ }^{3} \mathrm{MA}$ Aldred, ${ }^{1} \mathrm{CL}$ Shovlin. ${ }^{1}$ Imperial College London, London, UK; ${ }^{2}$ London North West University Healthcare NHS Trust, London, UK; ${ }^{3}$ Indiana University School of Medicine, Indianapolis, USA

\subsection{6/thorax-2021-BTSabstracts. 101}

Introduction and Objectives Pulmonary arteriovenous malformations (PAVMs) are most commonly caused by hereditary haemorrhagic telangiectasia (HHT). This multisystemic condition, inherited as an autosomal dominant trait, results from a heterozygous loss-of-function variant in ACVRL1, ENG or SMAD4. Heterozygous endothelial cell phenotypes have proved elusive, hindering preclinical testing of potential therapeutic agents. Here, our objective was to define the transcriptional changes occurring in patient-derived blood outgrowth endothelial cells (BOECs).

Methods With ethical approvals (16/ES/0095), BOECs were established from HHT/PAVM patients heterozygous for a pathogenic variant in ACVRL1, ENG or SMAD4, and from heathy volunteers. HHT gene protein production by patient and control BOECs was evaluated by ${ }^{35} \mathrm{~S}$-methionine pulse chase experiments. Single cell qRT-PCR aimed to verify expression and heterogeneity of 48 transcripts in 40 viable (DRAQ7 negative) BOECs per genotype. Long and short RNA libraries were generated from BOECs prior to Illumina HiSeq sequencing of paired-end reads, and alignment to GRCh38. Differential alignments were used to rank transcripts for discovery gene ontology process identifications.

Results 24 BOEC lines were established from patients heterozygous for one of 10 different nonsense (stop gain) pathogenic variants in ENG, ACVRL1 and SMAD4, with a median of two donors per genotype. Pulse chase experiments distinguished the genotypes. Blinded analyses of normalised RNASeq alignments also identified the source heterozygous HHT genotypes: ENG alignments were lowest in heterozygous $E N G^{+/-}$BOECs (Dunn's p=0.0089); ACVRL1 alignments lowest in heterozygous ACVRL1 $1^{+/}$BOECs $(\mathrm{p}=0.0040)$ and SMAD4 alignments lowest in heterozygous SMAD4 ${ }^{+/-}$BOECs $(\mathrm{p}=0.007)$. By single cell qRT-PCR, $7 / 48$ (15\%) genes were expressed in all BOECs, 7/48 (15\%) in no BOECs with 34 genes expressed in a proportion of BOECs. Seven genes displayed differential expression patterns between HHT and control BOECs, confirmed by distribution plots of all 16,807 RNASeq Ensembl transcript alignments in BOECs from different donors. Ranking transcripts by differential alignments in ENG/ACVRL1/SMAD4 compared to control BOECs, identified consistent gene ontology processes enriched compared to equivalent numbers of randomly-selected transcripts.

Conclusions There are reproducible, transcriptional signatures in pulmonary AVM and HHT patient-derived BOECs distinguishable from healthy volunteer BOEC signatures. Common patterns for ACVRL1, ENG and SMAD4 BOECs suggest a shared HHT transcriptome phenotype.

\section{S96 PULMONARY ARTERIOVENOUS MALFORMATIONS - GENETIC VERSUS CLINICAL EVIDENCE OF UNDERLYING HEREDITARY HAEMORRHAGIC TELANGIECTASIA}

${ }^{1} \mathrm{~L}$ Sharma, ${ }^{2} \mathrm{~A}$ Alsafi, ${ }^{2} \mathrm{~T}$ Ferguson, ${ }^{2} \mathrm{~J}$ Redhead, ${ }^{2} \mathrm{WL}$ Genomic Medicine Centre, ${ }^{1} \mathrm{CL}$ Shovlin. ${ }^{1}$ Imperial College London, London, UK; ${ }^{2}$ Imperial College Healthcare NHS Trust, London, UK

\subsection{6/thorax-2021-BTSabstracts.102}

Introduction and Objectives Pulmonary arteriovenous malformations (PAVMs) result in early onset but preventable strokes and other complications. Patients know that PAVMs can be a familial condition, most commonly due to hereditary haemorrhagic telangiectasia (HHT). Since the April 2020 NHS National Genomic Test Directory launch, patients with PAVMs are eligible for gene testing only if they already meet a definite clinical diagnosis of HHT, requiring two further Curaçao Criteria from nosebleeds, mucocutaneous telangiectasia, or first-degree relative with HHT. Our goal was to test the validity of this requirement.

Methods We audited ClinVar-listed variants in the major HHT genes, and with ethical approval, case notes of patients with PAVMs who had undergone NHS genetic testing. Tests were ordered predominantly between 2015-2019 through Mainstreaming Genomics initiatives via gene-test panels, or whole genome sequencing through the 100,000 Genomes Project.

Results ClinVar lists 2,804 variants in ENG, ACVRL1 and SMAD4, including 909 likely pathogenic/pathogenic variants that diagnose HHT. Most are loss-of-function frameshift, stopgain and splice site variants (390/645 [60\%] for ENG/SMAD4; 126/264 [47\%] for ACVRL1). At least 50\% of people with one of these variants would be expected to have PAVMs. At our institution, 124 patients with PAVMs were the first in their family to have a gene test. Of these, 83 (67\%) tested positive for HHT, i.e. were found to be heterozygous for a likely pathogenic or pathogenic variant in ENG, ACVRL1 or SMAD4. Focussing on the 83 patients with PAVMs and genetically-diagnosed HHT, only 63/83 (76\%) met three or more Curaçao criteria. For the remaining 20 patients with PAVMs and a positive HHT gene test, none met the family history criterion for HHT. While 14 (70\%) described nosebleeds as an adult, only $3(15 \%)$ had classical HHT telangiectasia, and the cohort included families where pulmonary AVMs (single or multiple) were the only HHT clinical feature across individuals with ENG pathogenic variants in two generations.

Conclusions There is a high burden of deleterious variants in HHT genes. Two-thirds of unselected PAVM patients have genetically-confirmed HHT, but of these, 1 in 4 display few if any clinical features of HHT. Wider gene testing is recommended.

\section{S97 INVESTIGATING THE PRO-FIBROTIC EFFECTS OF GALECTINS IN IPF - A POTENTIAL ROLE FOR GLYCAN- MEDIATED INTERACTIONS WITH INTEGRINS}

\footnotetext{
${ }^{1,2} \mathrm{JF}$ Calver, ${ }^{2} \mathrm{G}$ Harris, ${ }^{1,2} \mathrm{RM}$ Lithgo, ${ }^{3} \mathrm{R}$ Slack, ${ }^{1,2} \mathrm{DJ}$ Scott, ${ }^{4} \mathrm{RG}$ Jenkins, ${ }^{4} \mathrm{AE}$ John. ${ }^{1}$ University of Nottingham, Nottingham, UK; ${ }^{2}$ Research Complex at Harwell, Oxford, UK; ${ }^{3}$ Galecto Biotech, Copenhagen, Denmark; ${ }^{4}$ Margaret Turner Warwick Centre for Fibrosing Lung Disease, NHLI, Imperial College London, London, UK
}

\subsection{6/thorax-2021-BTSabstracts. 103}

Introduction Integrins are a family of transmembrane heterodimer proteins differentially expressed on the cell surface of many lung cell types. Integrin-mediated activation of the key 